\title{
Electrode catheter ablation for ventricular tachycardia: efficacy of a single cathodal shock ${ }^{\star}$
}

\author{
RICHARD N W HAUER, ETIENNE O ROBLES DE MEDINA, PAUL J KUIJER, \\ PIETER W WESTERHOF \\ From the Department of Cardiology, Heart-Lung Institute, University Hospital Utrecht, and the \\ Interuniversity Cardiology Institute Netherlands, Utrecht, The Netherlands
}

SUMMARY Electrode catheter ablation was used to treat 11 distinct types of sustained ventricular tachycardias in eight patients. Rigid electrophysiological criteria were used to identify five left and five right ventricular arrhythmogenic sites; one of them gave rise to tachycardia with two distinct configurations. A single R-wave-synchronised 250 or $150 \mathrm{~J}$ cathodal shock was delivered at each site. One patient had mildly symptomatic episodes of sustained ventricular tachycardia during the first four days after the shock-there were no other complications. At discharge none of the patients was taking antiarrhythmic drugs. They were followed for 8-20 months (mean 14). Ablation abolished five of the 11 ventricular tachycardias. There was no recurrence in three of the eight patients. In two patients identical ventricular tachycardias recurred because the identification of the arrhythmogenic site was incorrect.

\begin{abstract}
Ablation of arrhythmogenic sites (origin of tachycardia) by a DC shock given by a catheter electrode is an attractive alternative for the treatment of ventricular tachycardia that is refractory to drugs. ${ }^{1-3}$ Multiple high energy shocks were used in several clinical studies to enhance the efficacy by disrupting alternative pathways in an extended arrhythmogenic area. ${ }^{134}$ But death and serious complications were reported and were related to the high energy used. We evaluated the efficacy of a single cathodal shock to the presumed arrhythmogenic site.
\end{abstract}

\section{Patients and methods}

\section{PATIENTS}

We used the following criteria to select appropriate candidates for ablation: (a) presence of spontaneous episodes of symptomatic uniform sustained ventricular tachycardia; (b) refractoriness to or

Requests for reprints to Dr Richard N W Hauer, Department of Cardiology, Heart-Lung Institute, University Hospital Utrecht, Catharijnesingel 101, PO Box 16250, 3500 CG Utrecht, The Netherlands.

* Parts of this article were presented at the 35th Annual Scientific Session of the American College of Cardiology, March 1986, Atlanta, Georgia.

Accepted for publication 13 June 1988 intolerance to at least three antiarrhythmic drugs; (c) without accepted indications for surgical treatment; (d) only one or two predominant distinct configurations of ventricular tachycardia during spontaneous episodes when the patient was not taking antiarrhythmic drugs; (e) identification of the arrhythmogenic site(s) according to rigid criteria (see below).

\section{ENDOCARDIAL CATHETER MAPPING}

Details of endocardial catheter mapping have been described previously. ${ }^{6}$ A new $6 \mathrm{~F}$ quadripolar USCI catheter (interelectrode distance $0.5 \mathrm{~cm}$ ) for mapping and shock delivery was introduced into either the femoral artery or vein and advanced to the left or right ventricular cavity. Recording of two bipolar electrograms derived from the distal and proximal pairs of electrodes could be obtained simultaneously with the four unipolar electrograms from these electrodes. Filters were set at $50-1000 \mathrm{~Hz}$ for the bipolar recordings and at $0 \cdot 1-1000 \mathrm{~Hz}$ for unipolar recordings. The aortic pressure was monitored during the entire mapping and ablation procedure via the side channel of the arterial introducer.

We tried to identify the arrhythmogenic site of each distinct ventricular tachycardia. Criteria for identifying the configuration are given in a previous article. $^{6}$ 
CRITERIA FOR ELECTROPHYSIOLOGICAL IDENTIFICATION OF THE ARRHYTHMOGENIC SITE

We used the following rigid criteria: (a) during extensive activation mapping of ventricular tachycardia the earliest local distinct diastolic potential (index potential) was consistently recorded each consecutive cardiac cycle; (b) the relation of this index potential to the subsequent QRS complex of ventricular tachycardia was constant during spontaneous or pacing induced change of the interval between consecutive index potentials; $(c)$ the index potential was absent during normal sinus rhythm but suddenly appeared before the first QRS complex of a distinct ventricular tachycardia; (d) the index potential suddenly disappeared or changed its configuration after the last QRS complex showing the configuration of ventricular tachycardia.

The second, third, and fourth criterion indicate a causal relation between the index potential and the subsequent QRS complex of the tachycardia. The first criterion and at least one of the other criteria were regarded as essental for the identification of the arrhythmogenic site. If the QRS configuration was identical during ventricular tachycardia and during pacing this was regarded as additional evidence.

\section{ELECTRICAL CATHETER ABLATION}

The catheter electrode at the arrhythmogenic site from which the most prominent unipolar index potential was recorded was subsequently connected to a switchbox. This permitted recording to continue or connected the area to the cathodal output of a standard defibrillator (Datascope M/D 2). The characteristics of this device are: Capacity $40 \mu \mathrm{F}$, value of the inductor $36 \mathrm{mH}$, internal resistance of the inductor $10 \Omega$. The anodal sink of the defibrillator was connected to a large external chest patch. This patch was positioned under fluoroscopic control, placing the target tissue between the cathode and anode.

Then the patient was sedated with intravenous etomidate. The defibrillator was charged and during stable recording of the index potential the appropriate catheter electrode was switched to the cathodal output. Immediately afterwards the defibrillator was discharged (sinusoidal $5 \mathrm{~ms}$ pulse) synchronously with the QRS complex. Only a single shock was delivered to each arrhythmogenic site. The energy delivered during each shock was approximately $250 \mathrm{~J}$ (stored energy $300 \mathrm{~J}$ ) with the exception of two shocks delivered to the thin right ventricular wall where approximately $150 \mathrm{~J}$ (stored energy $200 \mathrm{~J}$ ) was used. During delivery of the shock the right ventricular apex and the $H$ is bundle catheter were left in situ. Afterwards the patients were monitored in the catheterisation laboratory for about one hour. Prophylactic anticoagulant treatment was started and continued for one month. Antiarrhythmic drugs were not used.

\section{TISSUE DAMAGE AND EVALUATION OF HAEMODYNAMIC AND ARRHYTHMOGENIC EFFECTS}

The peak concentration of creatine kinase $\mathrm{MB}$ isoenzyme was measured by taking blood samples every two hours for up to 12 hours after the shock. The normal value in our laboratory was $<5 \mathrm{U} / 1$. Technetium-99 pyrophosphate scans for left ventricular ejection fraction measurements were performed before and two weeks after ablation. Patients were kept in the monitoring area and had continuous two channel Holter recording during the first week after the shock. They performed a treadmill exercise test (Bruce protocol) at the end of the monitoring period and when they were discharged they were not taking any antiarrhythmic drugs.

Table 1 Patients and characteristics of spontaneous ventricular tachycardias

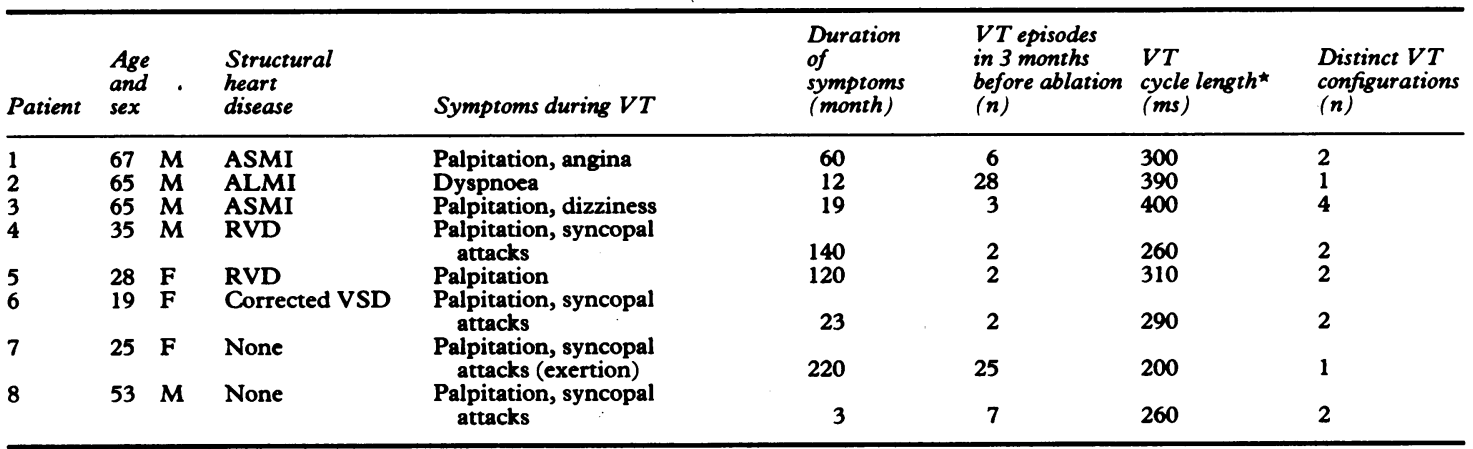

* Mean cycle length of most rapid ventricular tachycardia. ALMI, previous anterolateral myocardial infarction; ASMI, previous anteroseptal myocardial infarction; RVD, right ventricular dysplasia; VSD, ventricular septal defect; VT, ventricular tachycardia. 
Table 2 Catheter mapping and ablation data

\begin{tabular}{|c|c|c|c|c|c|c|c|}
\hline Patient. & $\begin{array}{l}V T \\
\text { considered } \\
\text { for ablation }\end{array}$ & $\begin{array}{l}\text { Presumed site of origin } \\
\text { (ablation site) }\end{array}$ & $\begin{array}{l}D A-Q R S \\
(m s)\end{array}$ & $\begin{array}{l}\text { Delivered } \\
\text { energy }(J)\end{array}$ & $\begin{array}{l}\text { Catheter } \\
\text { electrode } \\
\text { used } \\
(1=\text { tip } \\
\text { electrode })\end{array}$ & $\begin{array}{l}C K-M B \\
(U / l)\end{array}$ & Efficacy \\
\hline $\begin{array}{l}1 \\
2 \\
3 \\
4 \\
5 \\
\\
6 \\
7 \\
8\end{array}$ & $\begin{array}{l}\mathbf{V T _ { 1 }} \\
\mathbf{V T} \\
\mathbf{V T} \\
\mathbf{V T} \\
\mathbf{V T} \\
\mathbf{V T} \\
\mathbf{V T} \\
\mathbf{V T} \\
\mathbf{V T} \\
\mathbf{V T} \\
\mathbf{V T} \mathbf{T}_{1}\end{array}$ & $\begin{array}{l}\text { LV anteroseptal } \\
\text { LV anterior } \\
\text { LV anteroseptal } \\
\text { RVOT septal } \\
\text { RV anterior } \\
\text { RV apex } \\
\text { RV apex (same site) } \\
\text { RVOT } \\
\text { RVOT anterior } \\
\text { LV posterolateral† } \\
\text { LV anterolateral }\end{array}$ & $\begin{array}{c}75 \\
130 \\
110 \\
35 \\
25 \\
20 \\
5 \\
90 \\
5^{\star} \\
5 \dagger \\
5\end{array}$ & $\begin{array}{l}250 \\
250 \\
250 \\
250 \\
150 \\
150 \\
250 \\
250 \\
250 \\
250\end{array}$ & $\begin{array}{l}3 \\
4 \\
4 \\
2 \\
1 \\
1 \\
\\
1 \\
1 \\
1 \\
1\end{array}$ & $\begin{array}{r}42 \\
7 \\
8 \\
38 \\
22 \\
33 \\
\\
9 \\
30 \\
79 \\
103\end{array}$ & $\begin{array}{l}+ \\
+ \\
+ \\
+ \\
+ \\
+ \\
\pm \\
\pm \\
-\end{array}$ \\
\hline
\end{tabular}

CK-MB, peak concentration of creatine kinase MB isoenzyme; DA-QRS, interval between local diastolic activation (index potential) and onset of subsequent $Q R S$ complex at presumed site of origin; $L V$, left ventricle; $R V$, right ventricle; $R V O T$, right ventricular outflow tract; VT, ventricular tachycardia.

ॠActual site of VT origin was $1.5 \mathrm{~cm}$ from ablation site. DA-QRS at actual site of origin was $15 \mathrm{~ms}$.

†Actual site of VT origin was close to ablation site. DA-QRS at actual site of origin was $20 \mathrm{~ms}$.

\section{FOLLOW UP}

All patients were followed in the outpatient clinic every month for the first three months and every two or three months afterwards. We paid special attention to recurrences of symptomatic arrhythmias and to late complications.

\section{Results}

\section{PATIENTS}

Table 1 summarises the clinical characteristics of eight consecutive patients. Sixteen spontaneously (clinical) tachycardias with different configurations were recorded. However, five of these were recorded very rarely and only when the patients were taking antiarrhythmic drugs. The other 11 tachycardia configurations were recorded at least once in patients who were not taking antiarrhythmic drugs. Programmed electrical stimulation initiated 21 patterns of tachycardia, including all 16 tachycardias mentioned in table 1 and five additional non-clinical arrhythmias.

\section{CATHETER MAPPING AND ABLATION}

Ten combined mapping and ablation procedures were performed. Six patients had a single procedure and two patients had a second procedure three weeks and three months after the first. Only one single shock was delivered during each procedure.

Table 2 summarises the data on catheter mapping and ablation of tachycardia. Patients never remained in ventricular tachycardia throughout the mapping procedure. Tachycardias were often terminated by catheter positioning. In addition, if a patient became haemodynamically intolerant to the arrhythmia, it was terminated by burst pacing. Each site of origin of a tachycardia was identified by activation mapping. In addition, six patients had pacing during sinus rhythm at the presumed site of origin. This showed an identical QRS pattern in six of eight tachycardia configurations. A slightly different pattern was recorded during pacing at the site of the two tachycardia configurations in patient 5.

The origin of five tachycardias in four patients was identified at various sites in the left ventricle. Positioning the catheter electrode at posterolateral and anterolateral sites was quite easy, whereas anteroseptal and anterior sites of interest could be reached only after extensive manipulation. In these cases a stable position was obtained only after looping the catheter in the apical area and bending its tip upwards. The other six tachycardias originated in the right ventricle-in the outflow tract in three. No particular difficulties were encountered during positioning in the right ventricle.

Extensive mapping showed the earliest local diastolic activation (75-130 ms before the onset of the QRS complex) in patients with a previous myocardial infarction and in the patient with a corrected ventricular septal defect (figs 1, 2, and 3). Diastolic potentials in patients with arrhythmogenic right ventricular dysplasia or with primary electrical heart disease were recorded much later in diastole ( 5 to $35 \mathrm{~ms}$ in front of the QRS complex).

The shock in patient 7 and one of the shocks in patient 8 were later discovered not to have been delivered to the site at which the earliest diastolic potential was recorded. Diastolic activation at that site occurred 10 and $15 \mathrm{~ms}$ earlier than at the ablation site (table 2).

Immediately after the shock, appreciable ST segment elevation was recorded in leads II, III, and V1 

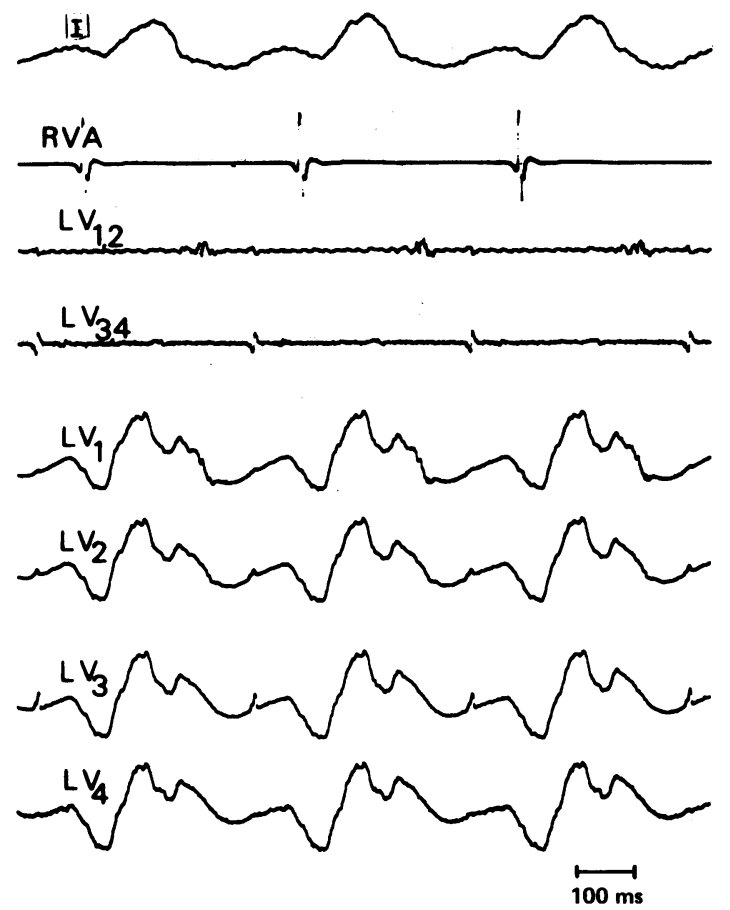

Fig 1 Recordings of a ventricular tachycardia during catheter mapping in patient 1. Figure shows the electrocardiographic lead I, the bipolar right ventricular apex electrogram ( $R V A)$, two bipolar electrograms ( $L V_{1,2}$ and $L V_{3,4}$ ) derived from the distal and proximal pairs of electrodes of the left ventricular mapping catheter, and four unipolar electrograms $\left(L V_{1}, L V_{2}, L V_{3}\right.$, and $\left.L V_{4}\right)$ derived from the left ventricular mapping catheter ( $L V_{1}$ derived from tip electrode). The most prominent unipolar diastolic potential (index potential) in $L V_{3}$ was recorded $85 \mathrm{~ms}$ ahead of the $R V A$ reference signal, or $75 \mathrm{~ms}$ before the onset of the $Q R S$ complex (the onset of $Q R S$ in $V_{1}$ (not shown here) was $10 \mathrm{~ms}$ ahead of the $R V A$ signal). Because a fixed relation between the index potential in $L V_{3}$ and the subsequent $Q R S$ complex was established by induced cycle change, the third electrode was considered to be positioned at the arrhythmogenic site and was chosen for delivery of a $250 \mathrm{~J}$ shock.

in patients 1 and 5 . ST segment depression in leads II and III was recorded in patient 7 . These changes in ST segment gradually diminished during the next few minutes. Atrioventricular conduction was not disturbed. Systolic aortic pressure was frequently below $90 \mathrm{~mm} \mathrm{Hg}$ immediately after the shock, but increased to the preshock value within minutes. Patients 7 and 8 felt a burning substernal chest pain after awaking, which responded promptly to intravenous droperidol and fentanyl.
TISSUE DAMAGE AND HAEMODYNAMIC EFFECTS Serum creatine kinase $M B$ isoenzyme reached its peak value of $37 \mathrm{U} / 1$ (range 7 to $103 \mathrm{U} / \mathrm{l}$ ) two to six hours after the shock (table 2). Table 3 shows the left ventricular ejection fraction measured before and after the last ablation procedure. There was a significant decrease of the ejection fraction in patient 4, who was treated with a shock to the right side of the septum, and in patient 8 , in whom two shocks were delivered at different left ventricular sites.

\section{ARRHYTHMOGENIC EFFECTS}

Episodes of non-sustained uniform ventricular tachycardia started three minutes after the shock in patient 7 (fig 4). During many recurrences, the QRS configuration was always the same, and identical to that seen before ablation. Extended Holter monitoring after ablation showed the continuation of
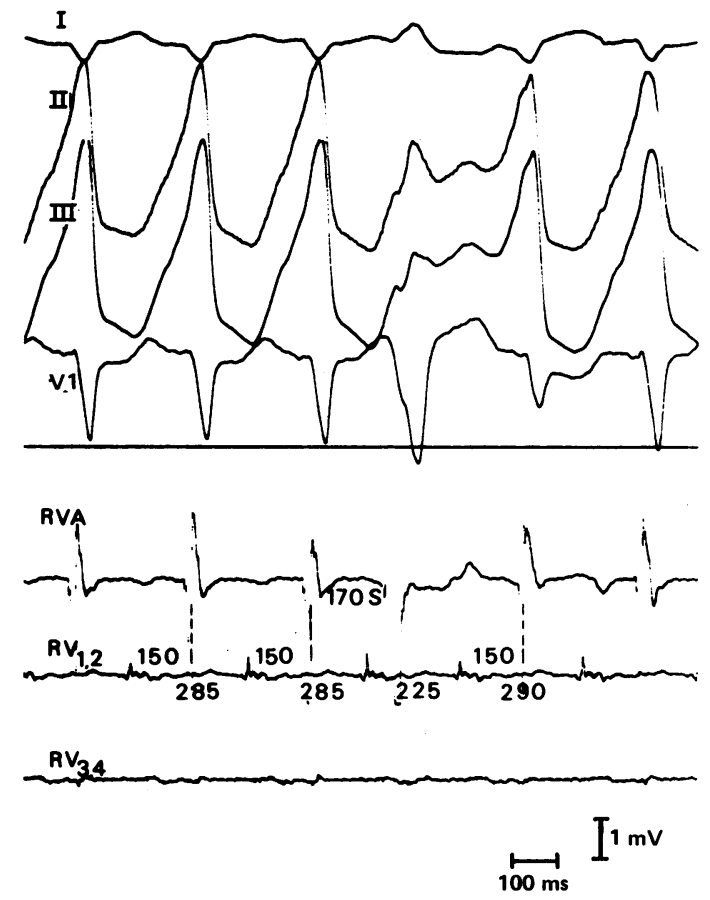

Fig 2 Recordings of a ventricular tachycardia in patient 6 during catheter mapping. The figure shows the electrocardiographic leads I, II, III and V1, the bipolar right ventricular apex electrogram ( $R V A)$, and two bipolar electrograms $\left(R V_{1,2}\right.$ and $\left.R V_{3,4}\right)$ derived from the distal and proximal pairs of electrodes of the mapping catheter, which was positioned at the presumed arrhythmogenic site in the right ventricular outflow tract. The index potential in $R V_{1,2}$ was recorded 150 ms ahead of the $R V A$ reference signal, or 90 ms before the onset of the QRS complex, and these intervals remain constant despite the change in cycle length induced by extrastimulus $(S)$. 

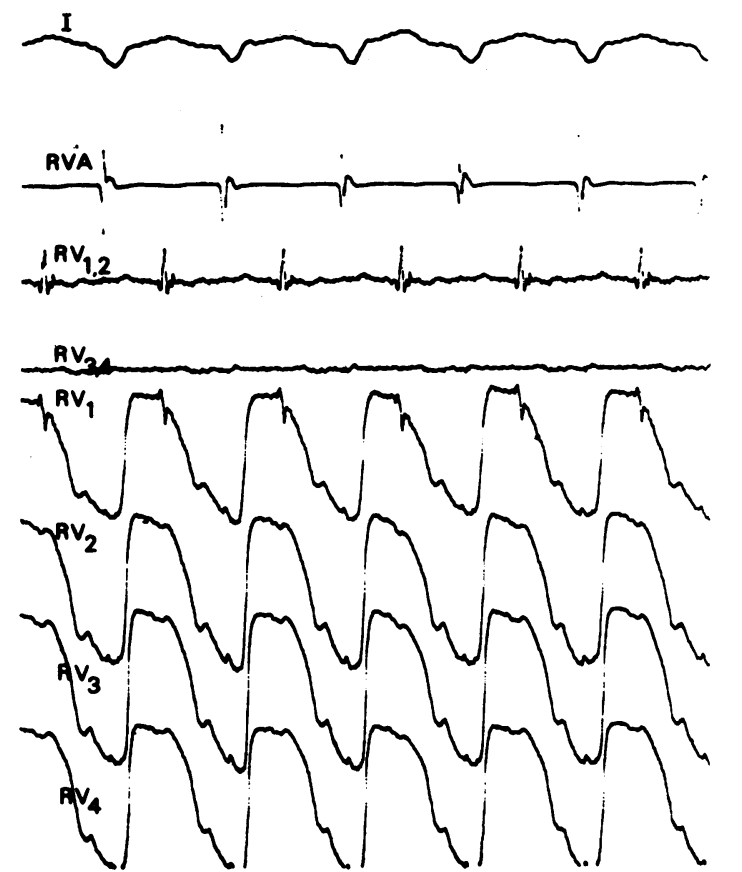

$\underset{100 \mathrm{~ms}}{\longmapsto} I^{2 \mathrm{mV}}$

Fig 3 Recordings of the same ventricular tachycardia as shown in fig 2 showing lead $I$, the bipolar right ventricular apex electrogram ( $R V A)$, two bipolar electrograms $\left(R V_{1,2}\right.$ and $R V_{3,4}$ ) derived from the distal and proximal pairs of electrodes, and four unipolar electrograms $\left(R V_{1}, R V_{2}, R V_{3}\right.$, and $R V_{4}$ ) derived from the electrodes of the mapping catheter ( $R V_{1}$ derived from the tip electrode). A distinct unipolar diastolic potential was recorded exclusively in $R V_{1}$, suggesting that only the tip electrode was in close contact with the ventricular wall at the site of origin of this ventricular tachycardia. This electrode was used to deliver a $250 \mathrm{~J}$ shock. Ablation failed. Compare with fig 1.

numerous episodes of uniform non-sustained and sustained ventricular tachycardia. The average cycle length of these tachycardias was about $\mathbf{4 0 0} \mathrm{ms}$. The minimal cycle length was $340 \mathrm{~ms}$. Figure 5 shows some characteristics of these episodes of tachycardia. Antiarrhythmic drugs were not used because these arrhythmias caused only mild symptoms (palpitation). The frequency of tachycardia rapidly declined on the 4th day. Findings were simlar in patient 4; however, the arrhythmias were asymptomatic in this patient.

EFFICACY

Table 2 shows the efficacy of ablation during the
Table 3 Left ventricular ejection fraction before and after ablation

\begin{tabular}{lll}
\hline & \multicolumn{2}{l}{ Ejection fraction } \\
\cline { 2 - 3 } Patient & Before ablation & After ablation \\
\hline 1 & 0.15 & 0.15 \\
2 & 0.13 & 0.14 \\
3 & 0.19 & 0.18 \\
4 & 0.50 & $0.41^{\star}$ \\
5 & 0.54 & 0.56 \\
6 & No data & No data \\
7 & 0.56 & 0.56 \\
8 & 0.50 & $0.41^{\star}$ \\
\hline
\end{tabular}

*Measured after second ablation.

follow up period. In patients 1,2 , and 5 there were no occurrences of symptomatic ventricular tachycardia during a follow up of 19,16 , and eight months,
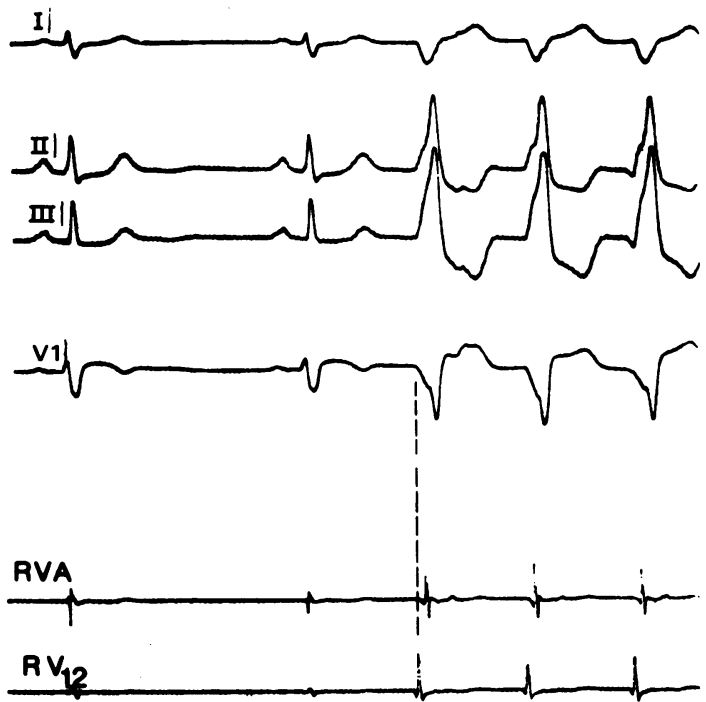

R $\mathbf{k}_{\mathbf{4}}$

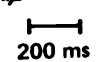

Fig 4 Onset of the first episode of ventricular tachycardia three minutes after a $250 \mathrm{~J}$ shock delivered to the arrhythmogenic site in the right ventricular outflow tract in patient 7. The cycle length was 980 ms during sinus rhythm and $440 \mathrm{~ms}$ during tachycardia. The coupling interval was $460 \mathrm{~ms}$. The first complex of the tachycardia had the same configuration as succeeding complexes, which was identical with the configuration of the ventricular tachycardia before ablation. The tip electrode of the mapping catheter (used for shock delivery) remained at the ablation site. The bipolar potential derived from the distal pair of electrodes of this catheter coincided with the onset of the surface $Q R S$ complex, suggesting that the tachycardia after ablation originated in the vicinity of the site of shock delivery. For abbreviations see fig 2 . 

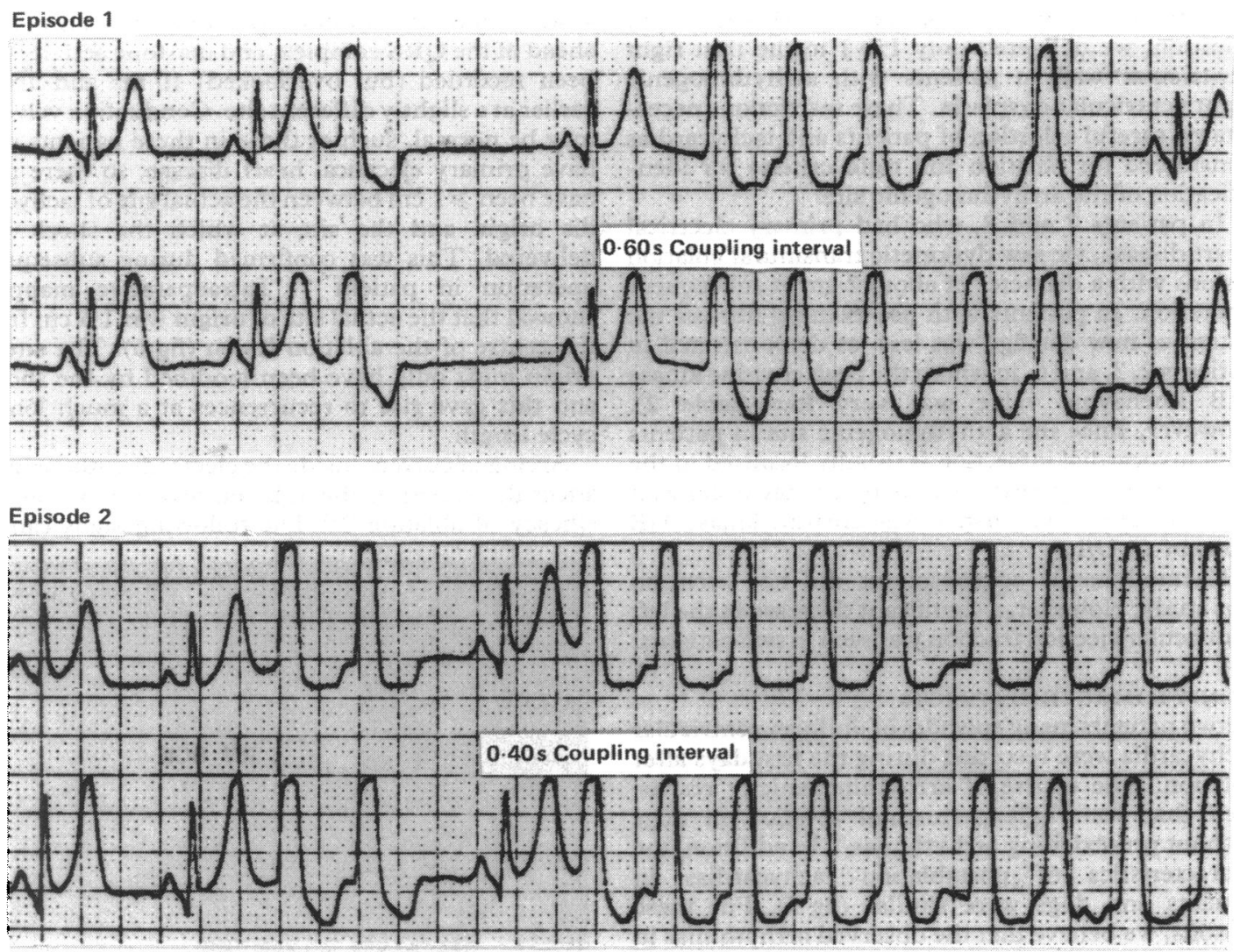

Fig 5 Some characteristics of two episodes of ventricular tachycardia recorded by two channel Holter monitoring in patient 7 after ablation. All tachycardia complexes had the same configuration during each episode. The coupling intervals were 0.60 and $0.40 \mathrm{~s}$, and the cycle length during tachycardia was also variable $(0.38-0.50 \mathrm{~s})$. Paper speed $25 \mathrm{~mm} / \mathrm{s}$.

respectively. In patient 5 a single $150 \mathrm{~J}$ shock successfully terminated two types of tachycardia.

Episodes of ventricular tachycardia before ablation in patient 7 were mostly related to exercise and were associated with many syncopal attacks. The cycle length varied from 270 to 200 ms. Episodes of nonsustained tachycardia with an identical configuration persisted after ablation. These arrhythmias, however, disappeared during exercise and the minimal cycle length increased to $330 \mathrm{~ms}$. Syncopal attacks did not recur. None the less, cryosurgery was performed four months after ablation because frequent recurrences of symptomatic arrhythmias (palpitations) did not respond well to drug treatment.

Symptomatic recurrences with similar configurations and cycle lengths have been documented in the other patients; in two of them additional drug treatment was effective.

Of the 10 configurations of ventricular tachycardia that were inducible with programmed electrical stimulation but were not considered for ablation, only one recurred during follow up (patient 3 ).

\section{Discussion}

\section{LOW VERSUS HIGH ENERGY}

Hypotension, pulmonary oedema, cardiac tamponade, and death have been reported after ablation with DC shock..$^{3-5}$ These complications seem to be related to $(a)$ the size and the number of ablation lesions (local effect) and (b) the height of the pressure wave during the shock (local and remote effects). Both factors are primarily energy dependent. ${ }^{7-9}$ In a previous experimental study a transmural lesion with a volume of $3.2 \mathrm{cc}$ was found one week after a single 250 J cathodal shock.

These observations prompted us to administer one single shock only at each arrhythmogenic site and to 
limit the total number of shocks in each patient. In addition, we delivered only $150 \mathrm{~J}$ to the thin right ventricular wall in patients with arrhythmogenic right ventricular dysplasia. These restrictions necessitated careful selection of patients and tachycardias considered for ablation and rigid criteria for identification of the arrhythmogenic site.

In patients 7 and 8 , who had primary electrical heart disease, we saw dyskinetic transmural ablation lesions with a diameter of about $2 \mathrm{~cm}$ at subsequent operation. In patients with pre-existing fibrosis the extent of new damage was less, as demonstrated in patients 2,3 , and 6 , in whom the peak creatine kinase MB isoenzyme value was very low (table 2). However, since the arrhythmogenic site in patients with myocardial infarction is usually localised in the border zone, more extensive damage may occur as it did in patient 1 who had a peak creatine kinase $M B$ value of $42 \mathrm{U} / 1$.

Haemodynamic complications did not occur in this study. However, a significant decrease in the left ventricular ejection fraction was seen in two patients.

\section{ARRHYTHMOGENIC EFFECTS}

In two patients many episodes of uniform ventricular tachycardia were recorded during the first days after ablation. The course of arrhythmogenicity resembled that seen in experimental ablation in dogs without pre-exisiting arrhythmias. ${ }^{10}$ In addition, the characteristics of postablation tachycardias in patients and dogs were similar (fig 5). For these reasons, we believe that the observed arrhythmias in both patients were caused by the arrhythmogenic effects of catheter ablation. These effects have also been reported by others. ${ }^{3511}$

\section{EFFICACY}

The success rate in our study is similar to the 33\% (29 of 88 patients) reported by the Percutaneous Cardiac Mapping and Ablation Registry. ${ }^{12}$ However, the mean stored cumulative energy reported to the Registry was $718 \mathrm{~J}$ and that used in our study was $280 \mathrm{~J}$.

In our study only one of 10 induced tachycardia configurations for which ablation was not considered recurred. This suggests that these arrhythmias were of limited clinical importance and that we had selected the appropriate tachycardias for ablation. These findings are at variance with those of Tonet et al who concluded from their study that all inducible sustained uniform ventricular tachycardias should be ablated. ${ }^{13}$

ANALYSIS OF FAILURES

In patients 7 and 8 there was a definite explanation for the failure of ablation. The shock was delivered at a site where the diastolic potential was only $5 \mathrm{~ms}$ ahead of the QRS complex, and diastolic activity had been recorded (but overlooked) $10 \mathrm{~ms}$ and $15 \mathrm{~ms}$ earlier at a slightly different site. Conduction velocity may be normal, such as those in these patients who have primary electrical heart disease, so there may have been $\geqslant 1 \mathrm{~cm}$ between the actual site of tachycardia origin and the site at which the shock was delivered. This was confirmed during subsequent operation in patient 7. Intraoperative mapping showed that the actual site of origin was $1.5 \mathrm{~cm}$ from the centre of the ablation lesion (fig 6). The site of origin must only have been modified by the shock, and this gave rise to recurrences at a much longer cycle length.

Arcing of current inside the electrode catheter may affect the extent of the ablation lesion and thus the efficacy of ablation. ${ }^{14} 15$ The following signs can be

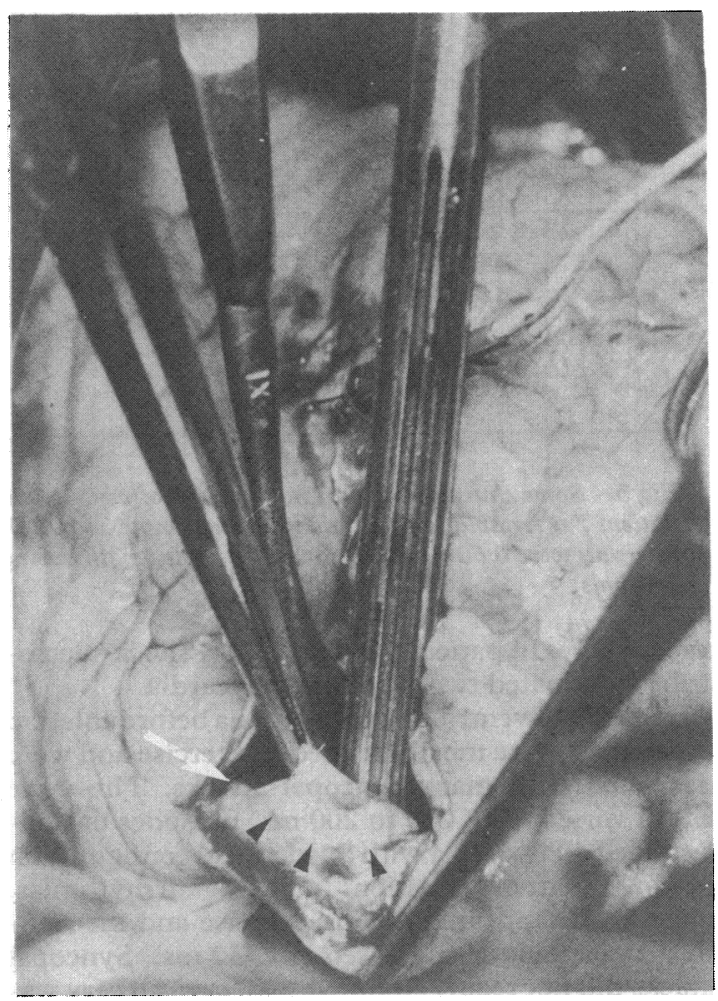

Fig 6 Ablation lesion in patient 7 seen during subsequent operation. An incision was made just apical of a transmural lesion in the anterior wall of the right ventricular outflow tract. The endocardial side of the lesion consisting of white scar tissue (black arrows) is clearly visible. The diameter of the lesion is about $2 \mathrm{~cm}$. Intraoperative mapping localised the origin of the ventricular tachycardia to an endocardial site $1.5 \mathrm{~cm}$ from the centre of the ablation lesion (white arrow). 
used to assess whether there has been current arcing: (a) recording of two or more identical unipolar electrograms derived from different electrodes, $(b)$ erosion of an electrode other than the one intended to deliver the shock, (c) damage to the insulation of the catheter, and (d) measurement of a change in the resistance. We found none of these signs in our study. It is possible, however, that the contact between the electrode and the ventricular wall may not have been optimal in all cases. ${ }^{16}$ Experimental data do not suggest that pre-existing fibrosis forms a barrier to lesion extension. ${ }^{16}$

Interpretation of mapping results remains difficult. A causal relation between the index potential and the subsequent QRS complex during tachycardia may be not enough to define the arrhythmogenic site. Alternative pathways are not excluded by this definition. Identification of these pathways and demonstration of a critical relation of the index potential to the next QRS complex may be necessary to enhance the success rate of ablation.

Our results indicate that catheter electrode ablation of the site of origin of ventricular tachycardia by a single cathodal shock, and without additional drug treatment, can be successful provided that the arrhythmogenic site has been correctly identified. We do not believe that all inducible tachycardias of all configurations require ablation. Catheter ablation itself can provoke ventricular tachycardia in the first days after the shock.

Further studies are needed to explain success or failure of electrode catheter ablation.

We thank Professor Frits L Meijler (Director of the Interuniversity Cardiology Institute Netherlands) for his support and encouragement; $\mathbf{M r}$ John $\mathrm{W}$ den Engelsman and Mrs Antony F Beukers for their interest and help with the Holter monitoring.

This study was supported by the Wijnand $M$ Pon Foundation, Leusden, The Netherlands.

\section{References}

1 Hartzler GO. Electrode catheter ablation of refractory focal ventricular tachycardia. J Am Coll Cardiol 1983;2:1107-13.

2 Scheinman MM. Catheter ablation for patients with cardiac arrhythmias. PACE 1986;9:551-64.

3 Belhassen B, Miller HI, Geller E, Laniado S. Transcatheter electrical shock ablation of ventricular tachycardia. J Am Coll Cardiol 1986;7:1447-55.

4 Tonet JL, Fontaine G, Frank R, Grosgogeat Y. Treatment of refractory ventricular tachycardias by endocardial fulguration [Abstract]. Circulation 1985;72 (suppl III):III-388.

5 Evans GT Jr, Scheinman MM, and the Executive Committee of the Registry. Catheter ablation of ventricular tachycardia foci: a report of the percutaneous cardiac mapping and ablation registry [Abstract]. Circulation 1986;74 (suppl II):II-460.

6 Hauer RNW, de Zwart MT, de Bakker JMT, et al. Endocardial catheter mapping: wire skeleton technique for representation of computed arrhythmogenic sites compared with intraoperative mapping. Circulation 1986;74:1346-54.

7 Hauer RNW, Straks W, Borst C, Robles de Medina EO. Electrical catheter ablation in the left and right ventricular wall in dogs: relation between delivered energy and histopathological changes. J Am Coll Cardiol 1986;8:637-43.

8 Bardy GH, Coltorti F, Ivey TD, et al. Some factors affecting bubble formation with catheter-mediated defibrillator pulses. Circulation 1986;73:525-37.

9 Holt PM, Boyd EGCA. Hematologic effects of the highenergy endocardial ablation technique. Circulation 1986;73:1029-36.

10 Hauer RNW, Robles de Medina EO, Borst C. Proarrhythmic effects of ventricular electrical catheter ablation in dogs. J Am Coll Cardiol 1987;10:1350-6.

11 Tonet JL, Baraka M, Fontaine G, et al. Ventricular arrhythmias during endocardial catheter fulguration of ventricular tachycardia [Abstract]. J Am Coll Cardiol 1986;7:236A.

12 Evans GT Jr, Scheinman MM, and the Executive Committee of the Registry. Catheter ablation for control of cardiac arrhythmias: a report of the percutaneous cardiac mapping and ablation registry. In: Breithardt, Borggrefe, and Lipes, eds. Nonpharmacological therapy of tachyarrhythmias. Mount Kisco, New York: Futura, 1987:243-57.

13 Tonet JL, Baraka M, Frank R, et al. Endocardial catheter fulguration of ventricular tachycardias: pitfalls of the clinical and nonclinical approach [Abstract]. Circulation 1985;72 (suppl III):III-388.

14 Fisher JD, Brodman R, Johnston DR. Non-surgical electrical ablation of tachycardias: importance of prior in vitro testing of catheter leads. $P A C E$ 1984;7:74-81.

15 Boyd EGCA, Holt PM. An investigation into the electrical ablation technique and a method of electrode assessment. $P A C E$ 1985;8:815-24.

16 Freericks MP, Hauer RN, Wittkampf FH, Bakker PF, Straks W, Robles de Medina EO. Determinants of lesion size after DC shock ablation: influence of electrode-wall contact and preexisting fibrosis [Abstract]. J Am Coll Cardiol 1988;11:178A. 